\title{
EVALUATION OF SIFT AND SURF FOR VISION BASED LOCALIZATION
}

\author{
Xiaozhi Qu, Bahman Soheilian, Emmanuel Habets, Nicolas Paparoditis \\ Universite Paris-Est, IGN, SRIG, MATIS, 73 avenue de Paris, 94160 Saint Mande, France \\ (firstname.lastname)@ign.fr
}

Commission III, WG III/4

KEY WORDS: Vision Based Localization, Local Bundle Adjustment, Feature Extraction, performance evaluation.

\begin{abstract}
:
Vision based localization is widely investigated for the autonomous navigation and robotics. One of the basic steps of vision based localization is the extraction of interest points in images that are captured by the embedded camera. In this paper, SIFT and SURF extractors were chosen to evaluate their performance in localization. Four street view image sequences captured by a mobile mapping system, were used for the evaluation and both SIFT and SURF were tested on different image scales. Besides, the impact of the interest point distribution was also studied. We evaluated the performances from for aspects: repeatability, precision, accuracy and runtime. The local bundle adjustment method was applied to refine the pose parameters and the 3D coordinates of tie points. According to the results of our experiments, SIFT was more reliable than SURF. Apart from this, both the accuracy and the efficiency of localization can be improved if the distribution of feature points are well constrained for SIFT.
\end{abstract}

\section{INTRODUCTION}

Localisation with Global Navigation Satellite Systems (GNSS) in dense urban areas suffers from masks of signals and multi-path errors and leads to significant errors. Dead reckoning methods using inertial navigation Systems (INS) were applied in order to reduce these errors by interpolating between GNSS interruptions. Vision based relative positioning methods such as visual odometry can provide the same improvement at lower costs.

Nowadays, a variety of methods have been proposed for vision based localization (Davison, 2003; Nistér et al., 2004; Mouragnon et al., 2006). The main steps of these methods can be summarized as: feature extraction, matching, pose estimation, and 3D structure reconstruction. The reliable matching constitutes the basis of vision based localization (Valgren and Lilienthal, 2010). The local feature based methods have been proved to be an excellent choice for pose estimation. However, the factors such as illumination changes, perspective deformations and moving objects make the matching a difficult task and influence the accuracy of pose estimation.

Thus the algorithms used for local feature extraction must be robust to these factors. The SIFT (Scale Invariant Feature Transform) which is invariant to scale change, rotation and illumination (Lowe, 2004) has been applied in the vision based localization (Se et al., 2001; Yang et al., 2009). However, the computation of SIFT feature extraction is time consuming. So a more efficient method called SURF (Scale Invariant Feature Transform), was proposed (Lowe, 2004). The SURF is also robust to the change of scale, orientation and illumination and is used for feature extraction in pose estimation methods (Murillo et al., 2007). In this paper, we chose SIFT and SURF as feature detector and descriptor. In particular, the performance of these two algorithms will be evaluated and compared.

In our localization approach, Local Bundle Adjustment (LBA) is applied to refine the pose parameters and 3D coordinates of tie points (Mouragnon et al., 2006). It is well known that the bundle adjustment is more precise than SLAM (Strasdat et al.,
2012), but it could become time consuming with the increasing quantity of images because of its high complexity. The LBA only process a fixed number of images at every step. Meanwhile, the propagation of uncertainty is considered from step to step (Eudes and Lhuillier, 2009).

Carefully designed experiments are performed to test the performance of SIFT and SURF for localization using ground truth image sequences captured by a Mobile Mapping System(MMS) called STEREOPOLIS (Paparoditis et al., 2012). In addition, the impact of image resolution is evaluated using sub-sampled images. The distribution of SIFT and SURF points is adapted using a grid adapter for point detection. Then the performance will be evaluated from following points of view:

- Stability: A relevant criterion to measure the stability of points extracted by the detectors is repeatability (Schmid et al., 2000), which is a ratio between the number of tie points and the detected points of interest in one image pair.

- Variance of points in image space: The variance of the observations can be evaluated by Variance Component Estimation (VCE) (Luxen, 2003).

- Accuracy of localization: This criterion can be estimated by comparing the estimated poses with the ground truth.

- Cost of computation: The time spent on every module of localization is also an important criterion for real time application.

This paper is organized as follows: Section 2. discusses the related work about the evaluation of feature detection. The section 3. introduces the SIFT, SURF, grid based extraction and the matching method for localization. In section 4., the localization approach is presented including estimation of initial values and the implementation of LBA. The evaluation criteria evaluation are defined in section 5.. Section 6. discuss our experiments' results. Conclusion and trends were presented in section 7 .. 


\section{RELATED WORK}

Many point detectors and descriptors are proposed in last years, so it is important to know the performance of each method that enables us to choose a suitable algorithm for feature extraction. We consider the performance of every method from several aspects. In order to explore the reliability and distinctiveness of the interest points, the repeatability criterion was defined by Schmid et al. (2000) . Similar strategies were also used to compare the performance of different descriptors and concluded that the SIFT descriptor outperformed other methods (Mikolajczyk and Schmid, 2005). However, SIFT is very time consuming (Grabner et al., 2006). Bay et al. (2008) proposed SURF point detector and descriptor which is faster than SIFT.

Many experiments have been designed to compare SIFT and SURF (Juan and Gwun, 2009; Khan et al., 2011; Saleem et al., 2012). The experimental results indicate that both SIFT and SURF are invariant for scale and orientation. But SIFT performs better than SURF in most of the cases on repeatability and robustness.

Most of the aforementioned evaluations are made in some image that capture the static scene. But in the operation of localization, the observed scene will be changed dynamically with the movement of vehicle or robot. In this case, it is more complex than the matching between images for a static scene. So the criterion used for the evaluation of the detector or descriptor performance would be different. A number of interest point detectors and feature descriptors were evaluated for robot navigation separately in (Schmidt et al., 2010). The SURF was applied as one of the feature description method and outperformed others by comparing the ratio of inlier matches. A similar strategy was token for the evaluation of interest point detectors and feature descriptors for visual tracking using in visual odometry . In particular, more detectors and descriptors were tested and the concept of repeatability was adopted (Gauglitz et al., 2011). The SIFT and SURF descriptors still performs better in this paper. Besides, the repeatability was also used to evaluate the detectors. SIFT and SURF still occupied the first and second places (Ballesta et al., 2007). Other new ways were combined to compare the performance of local feature detectors. In (Jiang et al., 2013), the state of the art of interest point detectors and feature descriptors were evaluated for stereo visual odometry in terms of three criteria that are repeatability, mean value of the back-projection errors and the absolute error of the image location.

Regarding the aforementioned references, the geometric reliability and distinctiveness of the interest points can be measured by the criterion of repeatability (Schmid et al., 2000). The precision of the feature points localization can be reflected by the backprojection error and accuracy of localization (Jiang et al., 2013). In this paper, the posterior variance of observations, estimated using VCE (Luxen, 2003) is used to represent the precision of the interest points detection. Apart from this, the impact of the interest points distribution and the resolution of image will also be investigated in this papers.

\section{FEATURE EXTRACTION AND MATCHING}

A brief introduction of SIFT and SURF algorithms are presented in section 3.1 and 3.2. In order to limit the number and distribution of the detected feature points, a grid based constraint is presented in 3.3. The matching method in localization is introduced in section 3.4 .

\subsection{SIFT}

SIFT has been widely used to extract the distinctive feature points and the algorithm can be summarized in four steps (Lowe, 2004). The first step is the creation of scale space and detection of potential key points. The scale space of image is built up using DoG (Difference of Gaussian) and the potential key-points are detected in the scale space. The second step is to compute the precise location and determine the scale of the key points in image scale space. In this step, the low contrast points will be filtered and the edge response of the key points will be eliminated to improve the stability. The third step is the orientation assignment. A histogram of the gradient directions in a region around key point is formed and the peak of the histogram is the dominant direction of the key point. In this case, the description of the key point can be made in relation to the dominant direction. Thus, it can achieve the invariance of image rotation. The last step is to create the descriptor. 128 bins which represent the gradient histogram values weighted by a Gaussian function, are extracted to describe the key points. In this paper, the OpenCV implementation of SIFT was used for detecting points and computing the corresponding descriptors.

\subsection{SURF}

In order to achieve the character of scale invariant for key points, the scale space of image is implemented for the detection of distinctive points. Lowe (2004) built the space using Gaussian pyramid layers of image and smoothed the sub-sampling images progressively to compute the DoG images. In SURF, the scale space is built up with box filter relaying on integral images which is much faster (Bay et al., 2008). Bay et al. (2008) detected the points of interest based on Hessian matrix. The dominant directions of the points of interest are calculated based on the Haar wavelet response in $x$ and $y$ direction with a small circular neighborhood. In order to describe the image feature around the interest points, a larger region centred on the points are extracted and oriented relative to the dominant direction. Then each region is split into $4 \times 4$ sub-regions. The feature is described by the Haar wavelet responses which are calculated at vertical and horizontal direction and weighted with a Gaussian factor. OpenCV implementation of SURF was used in this paper.

\subsection{Feature extraction with grid adapter}

Due to rich textures in street scenes, thousands of interest points are usually detected in one image. We accumulate the locations of interest points in image sequence, hence a density map is generated which indicates the probability of occurrence of interest points at the corresponding positions. Figure 1a depicts the density of SIFT points computed with 89 images. It is obvious that most of the points are distributed around the image center.

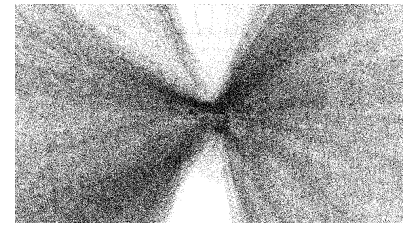

(a) SIFT

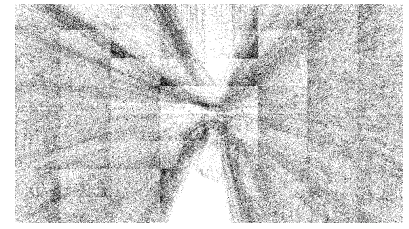

(b) SIFT+GRID
Figure 1 . The density maps of interest points.

Usually, the points near the image's center could be easily matched and tracked along the sequence. However, they are not useful in bundle adjustment. On the one hand, for the points situated at 
the center of images, the small parallax leads to inaccurate reconstruction of the points and consequently the poses parameters. On the other hand, the large number of points might decelerate the matching procedure and local bundle adjustment. A natural solution is in dividing the images into cells, then limiting the number of detected feature points in each cell. This kind of methods have been widely used to get well distributed feature points (Zhang et al., 1995; Kitt et al., 2010). In this paper, a $8 \times 8$ pixel grid is used that divide each image into 64 cells. In each cell, the maximum number of the feature points is fixed as 32 . Thus the most stable 32 feature points are kept if there are more than 32 feature points detected in one cell. If the number of detected feature points is less than 32, all of the points will be kept. The maximum number of the detected feature points is $64 \times 32$. The density of grid based SIFT is shown in figure $1 \mathrm{~b}$ where more uniform distribution of points is noticeable.

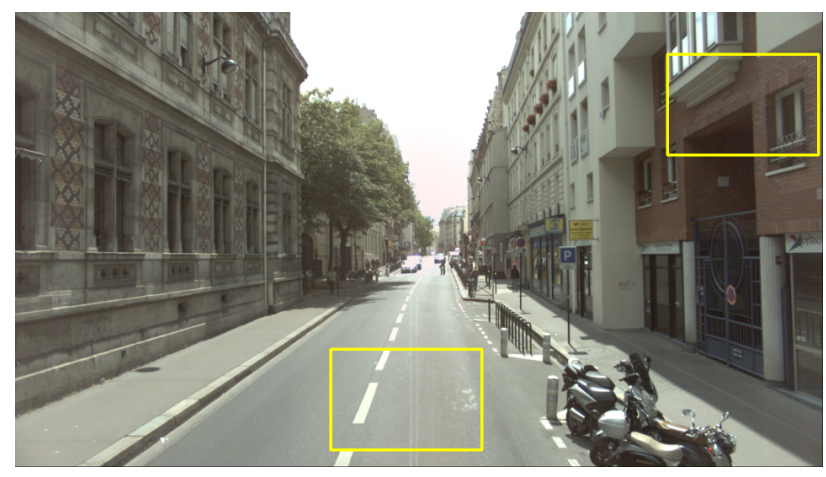

Figure 2. The interest points in the yellow rectangles are selected to show the difference from different extraction methods.

Figure 3 demonstrates the interest points on road and facades. The grid based methods enable to find more points in the noncentral regions of the images. The close-up patches are shown as the rectangles in figure 2.

\subsection{Matching}

In our localization system, the pose of every frame in the sequence is estimated, but only the key frames are kept, according to the moving distance. If the distance is larger than $0.5 \mathrm{~m}$, the frame will be taken into account as key frame. In the matching process., we do not search the interest points in current frame with the points in all the previous key frames. In contrast, only latest three key frames are matched with the current frame. $t$ is defined as the index for current image, the matching for image $t$ is to search the correspondences in the subsequence $(t-1, t-$ $2, t-3$ ). For each matching, the FLANN (Fast Library for Approximate Nearest Neighbors) (Muja and Lowe, 2009) is used to search the correspondences. The euclidean distance between two description vectors of feature points in two frames is employed to measure the similarity. The distance ratio $(T>0.6)$ is applied to filter the low quality matches. At last, the outliers are rejected using the epipolar constraint. An AC-RANSAC (A Contrario RANSAC) (Moulon et al., 2013) based algorithm is used to estimate the fundamental matrix. Reject the matching results that the distances from image points to its corresponding epipolar lines are larger than 2.0 pixel.

\section{THE PROCESS OF LOCALIZATION}

Our localization approach assumes that the pose of first frame and the distance from first to second frame are known in advance. Then we estimate the initial values for poses and some 3D object points. Finally, the LBA is applied to optimize these parameters.

\subsection{Initialization for image poses and object points}

We define $\left(R_{0}, C_{0}\right)$ as the pose of first image, where $R_{0}$ is rotation matrix from world to image and $C_{0}$ is the position of camera center in world coordinate system. $D$ is noted as the distance from first to second images. The pose of second image is estimated by:

$$
\left\{\begin{array}{l}
R_{1}=R R_{0} \\
C_{1}=-D \cdot R_{1} \nu
\end{array}\right.
$$

where, $(R, \nu)$ is the relative pose. The $R$ is rotation matrix and $\nu$ is the transformation from first to second image. We estimate $(R, \nu)$ using 5-point algorithm proposed by Nistér (2004). Then, some 3D object points are computed by triangulation using the matched tie points between first and second images and the poses of first and second image. From third frame, the pose will be estimated using a set of 3D-2D correspondences derived from the alignment between the interest points in new image and the reconstructed 3D object points. A novel P3P (perspective-threepoint) algorithm is used to solve the pose parameters (Kneip et al., 2011). The AC-RANSAC is employed to find the best estimates. With the newly estimated image pose, more 3D points can be reconstructed. The same procedure will be used to estimate the pose of other images and reconstruct more 3D points one by one.

\subsection{Local bundle adjustment}

Pose parameters are optimized by LBA (Mouragnon et al., 2006; Eudes and Lhuillier, 2009). Different with Global Bundle Adjustment (GBA) which aims to minimize the sum of squared backprojection errors (Triggs et al., 2000), LBA will minimize the back-projection errors under the constraint of prior poses $C_{p}^{0}$ in each sliding window, the cost function is shown in equation 3 .

$$
\begin{gathered}
\underset{C, X}{\operatorname{argmin}} \frac{1}{2}\left(v_{t}^{T} W_{t} v_{t}+v_{p}^{T} Q_{C_{p}}^{-1} v_{p}\right) \\
\left\{\begin{array}{l}
v_{p}=C_{p}-C_{p}^{0} \\
v_{t}=F\left(C_{p}, C_{n}, X_{t}\right)-m_{t}
\end{array}\right.
\end{gathered}
$$

where:

- $C_{n}$ : the new image poses in current processing window.

- $C_{p}$ : the prior poses inherited from previous steps.

- $X_{t}: 3 \mathrm{D}$ points.

- $F$ : projection function.

- $m_{t}$ : image points.

- $v_{t}$ : the vector of back-projection errors.

- $v_{p}$ : the vector of $C_{p}$ residuals.

- $W_{t}$ : the weight matrix for image points.

- $Q_{C_{p}}$ : the covariance matrix of $C_{p}$.

- $t$ : the index of frame.

In LBA, $C_{n}, C_{p}, X_{t}$ are parameters and $C_{n}=\left\{C_{t-n+1} \ldots C_{t}\right\}$ , $C_{p}=\left\{C_{t-N-1} \ldots C_{t-n}\right\}$. The $N$ is the size of sliding window and there are $n$ new images in $N$. The LBA is implemented with incremental approach, the procedure of the LBA method is also demonstrated in (Qu et al., 2015). In this paper, the CERES SOLVER (Agarwal et al., n.d.) is employed to resolve the least squares problem. 


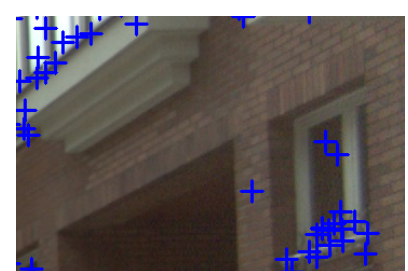

(a) SIFT

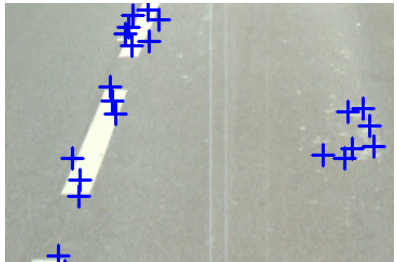

(e) SIFT

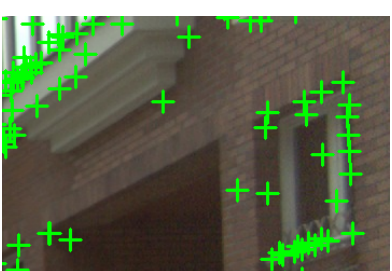

(b) SIFT+GRID

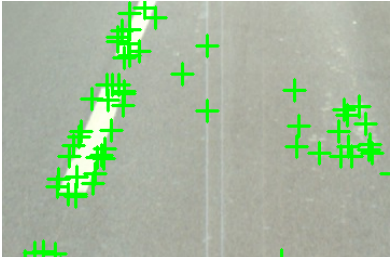

(f) SIFT+GRID

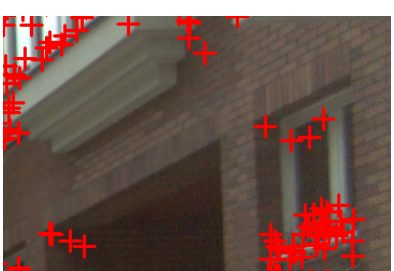

(c) SURF

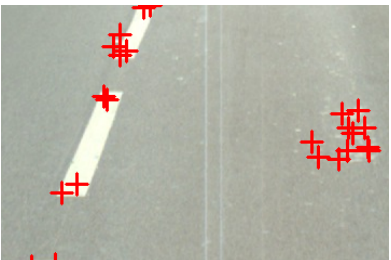

(g) SURF

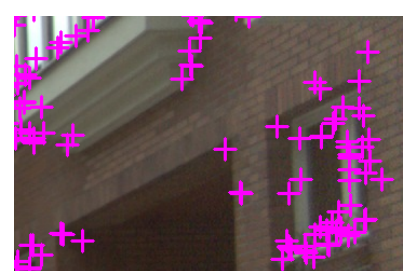

(d) SURF+GRID

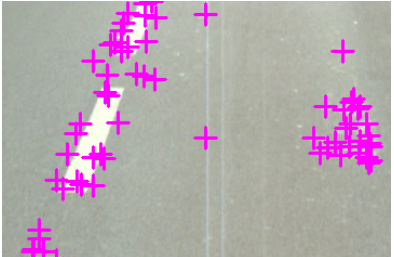

(h) SURF+GRID

Figure 3. Some interest points are removed on road and facade for SIFT and SURF detectors. But the grid based SIFT or SURF detectors are able to keep these useful points for localization.

\section{SETUP FOR THE EVALUATION}

\subsection{Repeatability}

The repeatability is defined by the ratio between the number of correctly matched point pairs and the number of detected feature points (Schmid et al., 2000). In one image pair $([i, j])$, the detected feature points are noted as:

$$
\left\{x_{i}^{k}\right\}_{k=1, \ldots, M_{i}},\left\{x_{j}^{m}\right\}_{m=1, \ldots, M_{j}}
$$

The correspondence between two points in the two images is noted as $x_{i}^{k} \underset{\epsilon}{\sim} x_{j}^{m}$ with respect to the condition of homography transformation:

$$
x_{i}^{k} \underset{\epsilon}{\sim} x_{j}^{m} \Rightarrow \operatorname{dist}\left(H \cdot x_{i}^{k}-x_{j}^{m}\right)<\epsilon
$$

where $H$ is homography matrix, $\epsilon$ is the radius of neighborhood. The number of correspondences between images $i$ and $j$ will be:

$$
\Phi(\epsilon)=\sum_{x_{i}^{k} \sim x_{j}^{m}} 1
$$

So the repeatability of interest points is defined as:

$$
\gamma=\frac{\Phi(\epsilon)}{\min \left(M_{i}, M_{j}\right)}
$$

The value of $\gamma$ provides a good measure of repeatability for planar scene in respect to homography transformation for one image pair. However, the matching in our localization system is a continuous approach that each new image is matched with previous three images. So the aforementioned measurement of repeatability can not be suitable for us. Therefore, we consider the ratio between the number of tie points to the the number of feature points in each image as a new measure of repeatability. Tie point set is defined as:

$$
\left\{x_{t}^{i} \mid \operatorname{dist}\left(F\left(C_{p}, C_{n}, X_{t}\right)-x_{t}^{i}\right)<\epsilon\right\}
$$

where $x_{t}^{i}$ is a tie point in current image $t, \epsilon$ is the threshold for back-projection error which is set as 3 pixels in this paper. The number of interest points of image $t$ is $M_{t}$, the repeatability corresponding to image $t$ is :

$$
\rho(t, \epsilon)=\frac{\left|\left\{x_{t}^{i} \mid \operatorname{dist}\left(F\left(C_{p}, C_{n}, X_{t}\right)-x_{t}^{i}\right)<\epsilon\right\}\right|}{M_{t}}
$$

In this paper, the mean values of $\rho(t, \epsilon)$ is calculated to measure the repeatability. We assume the number of images is $T$, so the repeatability for the feature detector is calculated by:

$$
\rho=\frac{1}{T} \cdot \sum_{t=0}^{t<T} \rho(t, \epsilon)
$$

\subsection{Posterior variance of interest points}

In our approach, we estimate the variance of the feature points using Variance Component Estimation method (VCE). The covariance matrix for the observations can be estimated by :

$$
Q=\sigma_{0}^{2} \cdot \widetilde{Q}
$$

where $Q$ is the variance matrix, $\sigma_{0}^{2}$ is the variance factor and $\widetilde{Q}$ is the initial variance matrix. We assume all the interest points have the same detecting precision for each detector and set $\widetilde{Q}$ as identity matrix. Thus, the variance for the images points is equal to $\sigma_{0}^{2}$. According to the theory of VCE, the variance factor $\sigma_{0}^{2}$ can be estimated by analyzing the residuals of the observations after bundle adjustment. The variance factor is estimated using the following equation (Luxen, 2003):

$$
{\hat{\sigma_{0}}}^{2}=\frac{\hat{v}_{t}^{T} \hat{v}_{t}}{r}
$$

where:

- $\hat{v}_{t}$ is the residual vector after adjustment.

- $r$ is the redundancy number which is equal to the difference between the number of observations and unknowns. 
LBA is an incremental approach and bundle adjustment is applied in a local processing window. In this case, the VCE should be employed for each processing step and a number of variance factors are estimated for one dataset. Apart from this, the prior estimates of images poses in LBA are also a kind of observations in processing step. This strengthen the difficulty of using VEC in LBA. Furthermore, the precision of one interest point detector should be same for one dataset. Thus, global bundle adjustment is the only option for VCE. The observations for GBA are the interest points, the unknowns are the image poses and $3 \mathrm{D}$ object points. The residuals after bundle adjustment are only related to image points. So the posterior variance of image points can guide the quality of image points detection.

\subsection{The accuracy of localization}

The goal of our approach is to evaluate the performance of SIFT and SURF for localization. So the accuracy of localization is one of the most important criterion. The final results of localization can be impacted by the precision of detector, the stability of feature points for matching and the distribution of the tie points. In order to make the criterion of localization accuracy comparable on trajectories with different lengths, the criterion is defined as the ratio between the absolute error of localization and the length of trajectory. The equation can be noted as:

$$
e_{t}=\frac{\left\|Z_{t}-Z_{t}^{0}\right\|}{L_{t}}
$$

- $Z_{t}$ is the estimated image location .

- $Z_{t}^{0}$ is ground truth.

- $L_{t}$ is the length of path from the beginning.

The numerator of equation 12 is the euclidean distance between the estimated position and ground truth. In this paper, the ground truth is acquired by a precise navigation system in our MMS.

\subsection{Running time}

For real time localization, the processing time is also an important criterion. We consider the running time from three aspects that are feature extraction, image matching and LBA. It is of couse the time for matching and LBA is highly related to the number of feature points. The computation time for LBA contains the time for initialization of the parameters and optimization.

\section{RESULTS AND ANALYSIS}

\subsection{Dataset design}

The data in this experiment were captured by STEREOPOLIS (Paparoditis et al., 2012). The ground truth for pose parameters is obtained by a precise navigation system. Images are captured by a calibrated front looking camera. The focal length of the camera is $10 \mathrm{~mm}$, the image size is $1920 \times 1024$ pixels. The FOV of the camera is $70^{\circ}$ in horizontal and $42^{\circ}$ in vertical. Four datasets are designed for evaluation which are noted as $D 1, D 2, D 3, D 4$. The difference between $D 1, D 2$ and $D 3, D 4$ is the average sampling distance. The sampling distance for first two sets $(D 1, D 2)$ is about $3 m$ but it is about $6 m$ for $D 3, D 4$. Table 1 shows the number of images and the length of each path.
Table 1. The number of images and the length of path for each dataset.

\begin{tabular}{c|c|c|c|c}
\hline dataset & $D 1$ & $D 2$ & $D 3$ & $D 4$ \\
\hline number of images & 91 & 89 & 87 & 106 \\
\hline length $(\mathrm{m})$ & 277 & 276 & 570 & 627 \\
\hline
\end{tabular}

The paths $D 1, D 2, D 3, D 4$ are shown in figure 4 .

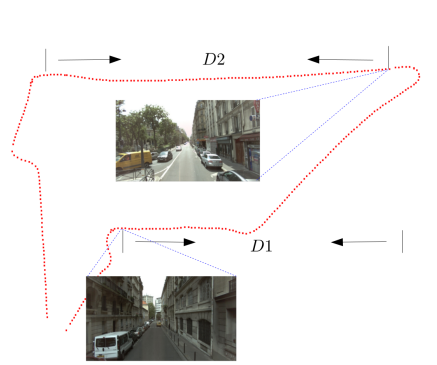

(a) $D 1, D 2$

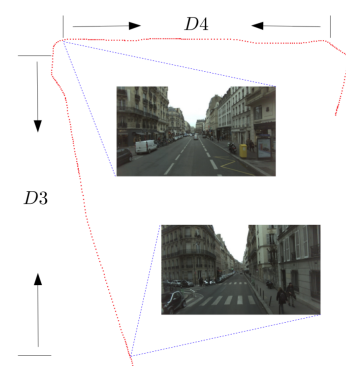

(b) $D 3, D 4$
Figure 4. The red dots are the locations of images and the images show the first view of the sequence.

To test the impact of the image resolution, a sub-sampled set is generated for every original image dataset. The sub-sampled image sets are generated by resizing the images from original size $(1920 \times 1024)$ to $960 \times 512$ pixels. We note the original image as HD (High Definition) image and the sub-sampled image as QHD (Quarter High Definition). It is obvious that the number of detected points will be reduced in QHD image, so that the speed of localization will be improved. Figure 5 shows a sample of HD and QHD image.

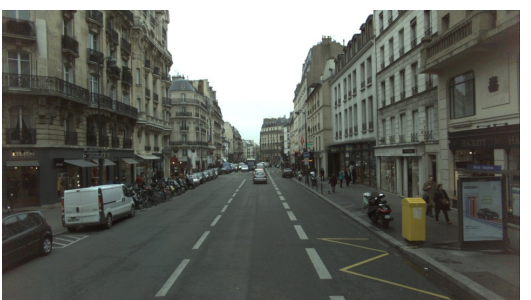

(a) HD image

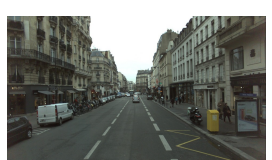

(b) QHD image
Figure 5. HD and QHD image.

\subsection{Evaluation measurements}

The SIFT, SURF, SIFT+GRID and SURF+GRID are used to extract the points of interest in HD and QHD images for every datasets. Thus, eight different types of results can be obtained for every datasets. Here the SIFT+GRID and SURF+GRID correspond to SIFT and SURF detectors applied with a grid adapter. The parameters for SIFT and SURF detectors are fixed for all the cases.

6.2.1 Repeatability of feature points: Figure 6a depicts the repeatability for the four datasets and the $\epsilon$ in equation 9 is set to 3.0 pixels. The $x$ axis corresponds to the applied method while the $y$ axis is the repeatability. There are no repeatability values for the QHD+SURF+GRID case in $D 3, D 4$ datasets. Because there are too few image points that are matched in some QHD image pairs when the SURF+GRID method is used for feature extraction for $D 3, D 4$. The reason is that the deformation caused by movement of images is heavier in $D 3, D 4$ because of the larger 
sampling distance. Therefore, it is more difficult to match the interest points in $D 3, D 4$. The previous investigation showed that SIFT outperforms SURF with the growing deformation caused by affine transformation (Juan and Gwun, 2009) between two images. So SIFT can still be used in QHD+GRID case while SURF are failed to get enough matches.

The comparison between SIFT and SURF is made under same conditions (same resolution, constraint). In most of cases, SIFT shows better rate than SURF. For instance, the repeatability for HD+SIFT case is higher than HD+SURF and HD+SIFT+GRID is higher than $\mathrm{HD}+\mathrm{SURF}+\mathrm{GRID}$.

Meanwhile, SIFT also shows its scale invariance. The repeatability rates are kept stable and even were improved a little bit from HD+SIFT to QHD+SIFT, but the repeatability for HD+SURF decreases when sub-sampled images were used.

In addition, the repeatability decreases where the grid adapters are applied. As we discussed before, the feature points around image center can be easily matched between images, because of the small displacement of the pixel in center region in comparison with the pixel in the region out of center part where the view point changes are larger. If we reduce the points distributed in this region, it also removes a large number of potential tie points. Compared with SURF, the SIFT provides better results. The decreasing for SIFT is smaller than SURF when grid adapter is used. Besides, SIFT would be a better option than SURF when process the large sampling distance image sequence. By observing the repeatability in HD+SIFT+GRID and HD+SURF+GRID, the repeatability values provided by SIFT for $D 1, D 2, D 3, D 4$ are similar while the values for $D 3, D 4$ using SURF are smaller.

6.2.2 Posterior variance of interest points: The posterior estimated variances of the feature points are shown in figure $6 b$. Because of the same reason as previous section, the variance for QHD+SURF+GRID cannot be estimated with VCE.

The results in figure $6 \mathrm{~b}$ tend to that SIFT still gives better performance than SURF. Because the variance for SIFT is smaller than SURF when they are used under same conditions. The variances for SIFT method for all tested cases are less than 0.5 pixel while the variance for SURF detector is around 0.5 pixel and even larger. Apart form this, the diagram also depicts that the precision of the feature points for original images is slightly better than sub-sampling image by comparing the variance values in HD and QHD images for SIFT and SURF.

6.2.3 Accuracy of localization: The maximum error of localization occurs at the last image in our approach and the values are shown in figure $6 \mathrm{c}$ for each method. The relative error of estimated image positions is calculated with the method mentioned in section 5. and the results are shown in figure $6 \mathrm{~d}$. The state of the art visual odometry method can approach $0.88 \%$ on the accuracy of image position in KITT benchmark using stereo images (Cvii and Petrovi, 2015). We achieve around 0.7\% (Fig 6d) using $\mathrm{HD}+\mathrm{SIFT}$ for all the four cases with monocular sequences. But we should mention that the resolution of our experimental images are higher than KITT datasets that the resolution is $1226 \times 370$ pixels.

The diagram depicts that the errors of position increase when the resolution of image changes from HD to QHD. Meanwhile, the grid adapter is not suitable for SURF especially for the large sampling distance cases $(D 3, D 4)$. Both the maximum and relative errors increase quickly if the grid adapter is used. The result in both diagrams also reflect the robustness of SIFT, the errors keep flat for all the cases related to SIFT. We find that the

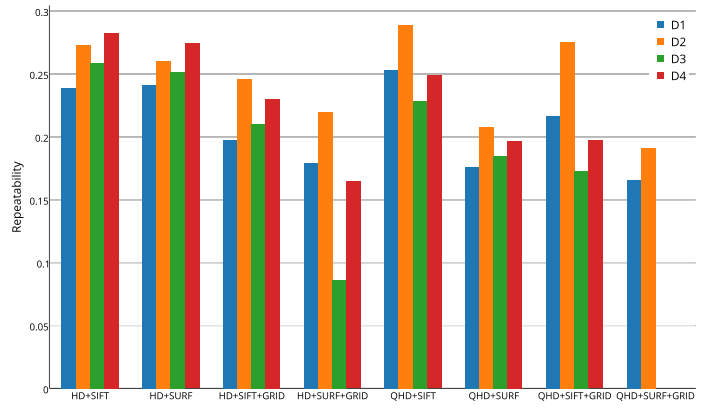

(a) Repeatability for each feature extraction method on different datasets.

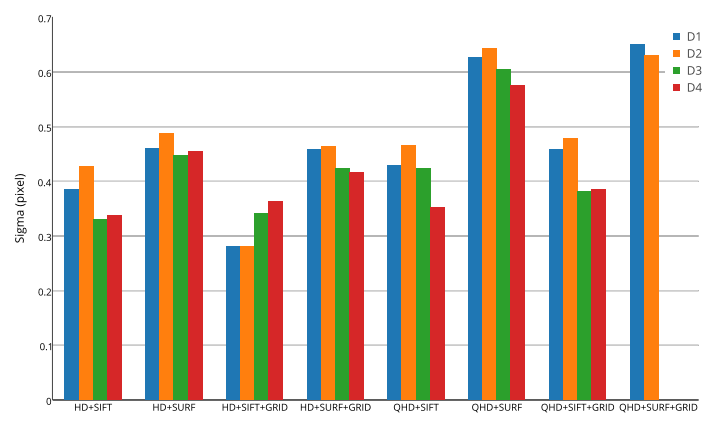

(b) The posterior variance of interest points.

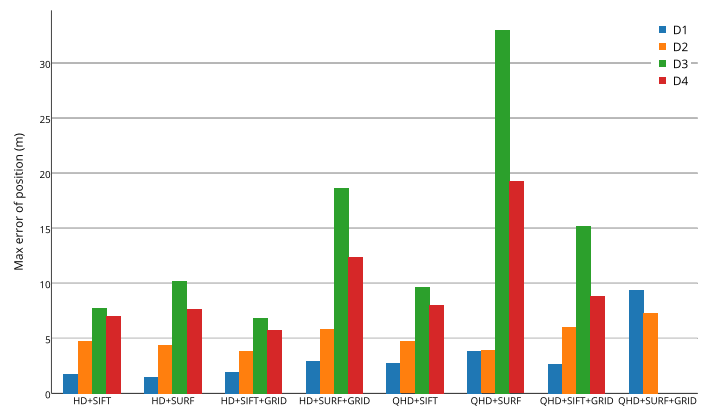

(c) The max errors of image position for different image sets. The lengths of path for $D 3, D 4$ are longer than $D 1, D 2$, so the maximal error of image position is large.

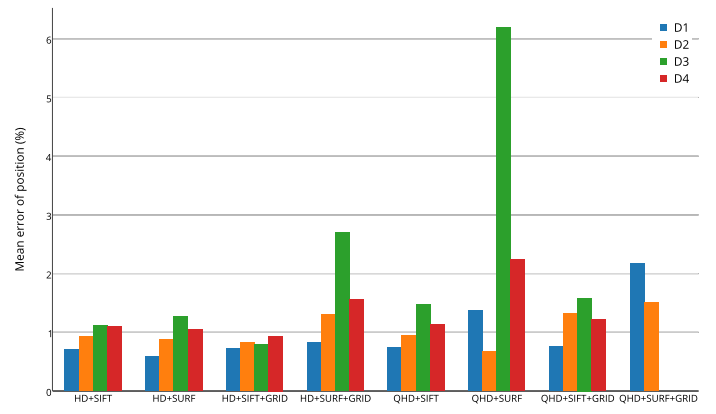

(d) The mean values of relative error for image position.

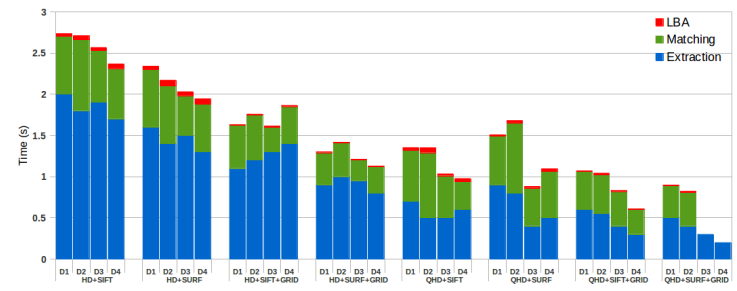

(e) The average processing time per image for feature extraction, matching and LBA.

Figure 6. The experiment results for different criterion. 
HD+SIFT+GRID is the most stable way among all the feature extraction strategies for localization, the relative error is less than one percent for $D 1, D 2, D 3, D 4$. Furthermore, HD+SIFT+GRID has more accurate localization results than HD+SIFT at most sets $(D 2, D 3, D 4)$. But this is not the case for SURF which leads to worse results when the grid adapter is applied. These figures also tend to illustrate the advantage of high resolution images where most of HD images can obtain better results than QHD with the same feature extraction method.

6.2.4 Runtime: Figure 6e shows the runtime for feature extraction, matching and LBA. Each part of localization procedure is demonstrated with different color of bin. The height of the bin represents the average processing time per image. It is obvious that the time spent on feature extraction and matching is much higher than LBA in our localization procedure from figure $6 \mathrm{e}$, where the blue and green bins are much higher than the red bins. In addition, the processing time can be reduced by subsampling the image (QHD) or limiting the number of feature points (GRID). However, a trade-off between efficient and accuracy should be found. The SIFT and SURF used in this experiment are standard CPU based implementation. In the future, the feature extraction part can be speed up with GPU.

\subsection{Further analysis}

An accurate localization usually obtains low posterior variance and high repeatability. If the repeatability is too low, the localization may not be accurate. For instance, the repeatability for D3 using HD+SURF+GRID is the very small (see Fig 6a), so the error of position is high in figure $6 \mathrm{~d}$.

Combining the results of repeatability, posterior variance and the accuracy, SIFT outperforms SURF on stability. For instance, the accuracy reduces too much when the resolution is decreased or the grid adapter is used, but SIFT performs better. In particular, the HD+ SIFT+GRID strategy achieves excellent results for all datasets, the posterior variances are almost the smallest (Fig 6b) in all the methods. Although the repeatability of HD+SIFT+GRID is slightly smaller than HD+SIFT, the localization accuracy is almost the best. This indicates that the distribution of tie points is a more important factor than the large quantity of tie points.

The posterior variance is relevant for evaluating the back projection errors of the tie points. Thus the final estimation of posterior variance for the image points would be influenced by the accuracy of localization and precision of image point detection. As we discussed before, the distribution of tie points also impact the localization accuracy. However, if the same strategy is used for feature extraction, the distribution of tie points is similar (cf. figure 7 which shows the accumulation map of HD+SIFT and HD+SURF). In this case, the posterior variance can reflect the precision of image points detector since the accuracies of the localization are equivalent. In figure $6 \mathrm{~d}$ and $6 \mathrm{c}$, the errors of HD+SIFT and HD+SURF are equivalent, but the posterior variance of SURF is larger than SIFT in figure $6 \mathrm{~b}$. This phenomenon indicates that the SIFT detector is more precise than SURF in OpenCV implementation.

The HD+SURF performs slightly better than HD+SIFT for localization accuracy. Even the distribution of the tie point for $\mathrm{HD}+\mathrm{SIFT}$ and HD+SURF is very similar in figure 7, the quantity of tie points in the out of center area for SURF is higher where the intensity in figure $7 \mathrm{~b}$ is darker than figure $7 \mathrm{a}$. So there might be more reliable tie points in bundle adjustment.

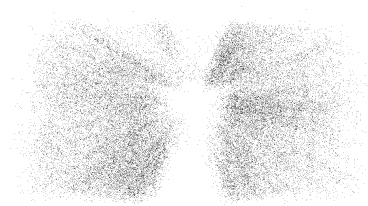

(a) $\mathrm{HD}+\mathrm{SIFT}$ (b) HD+SURF

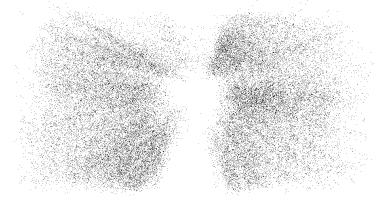

Figure 7. The maps of tie points for HD images in $D 1$. The image points are detected without GRID constraints.

\section{CONCLUSION}

An evaluation of SIFT and SURF for vision based localization was introduced in this paper. From our experiment, SURF was faster than SIFT on feature extraction, but SIFT was more reliable considering the results from repeatability, precision and the accuracy of estimated poses. Therefore, SIFT might be more suitable for our localization method. With the grid adapter, a better uniformly distributed interest points can be guaranteed. Thus we conclude that grid adapter (HD+SIFT+GRID) is the best option for us in localization approach.

In our experiments, the HD images have higher spatial resolution than QHD images. Theoretically, HD image should obtain better localization accuracy. However, the posterior variance for HD+SIFT+GRID is only slightly better than QHD+SIFT+GRID while the absolute errors of localization are equivalent, as shown in figure $6 \mathrm{~d}$ and $6 \mathrm{~b}$. This situation might mean that the tie point acquired from QHD+SIFT+GRID could be more stable and better distributed. But more investigation need to be done in this part. In order to take benefit from good distribution and precise 2D localization of tie points, one possible solution is to detect and match the interest points in QHD level, and re-localize the points in HD level. This kind of strategies have already been applied in some research(Bellavia et al., 2015). Besides, we should mention that some factors such as pose estimators, the triangulation precision would also influence the localization accuracy. In the future work, more detailed experiments should be considered to include all of these factors. More advanced computing technologies such as GPU, application-specific integrated circuits (ASIC) or fieldprogrammable gate arrays (FPGA), can be considered to speed up the process.

\section{ACKNOWLEDGE}

we gratefully thank Valérie Gouet-Brunet and David Vandergucht in MATIS Lab. for the discussions and suggestions about the feature extraction and matching.

\section{REFERENCES}

Agarwal, S., Mierle, K. and Others, n.d. Ceres solver. http: //ceres-solver.org.

Ballesta, M., Gil, A., Reinoso, O. and Mozos, O. M., 2007. Evaluation of interest point detectors for visual slam. International Journal of Factory Automation, Robotics and Soft Computing 4, pp. 86-95.

Bay, H., Ess, A., Tuytelaars, T. and Van Gool, L., 2008. Speededup robust features (surf). Computer vision and image understanding 110(3), pp. 346-359.

Bellavia, F., Fanfani, M. and Colombo, C., 2015. Selective visual odometry for accurate auv localization. Autonomous Robots pp. 1-11. 
Cvii, I. and Petrovi, I., 2015. Stereo odometry based on careful feature selection and tracking. In: Mobile Robots (ECMR), 2015 European Conference on, pp. 1-6.

Davison, A., 2003. Real-time simultaneous localisation and mapping with a single camera. In: Computer Vision, 2003. Proceedings. Ninth IEEE International Conference on, pp. 1403-1410 vol.2.

Eudes, A. and Lhuillier, M., 2009. Error propagations for local bundle adjustment. In: Computer Vision and Pattern Recognition, 2009. CVPR 2009. IEEE Conference on, IEEE, pp. 2411-2418.

Gauglitz, S., Höllerer, T. and Turk, M., 2011. Evaluation of interest point detectors and feature descriptors for visual tracking. International journal of computer vision 94(3), pp. 335-360.

Grabner, M., Grabner, H. and Bischof, H., 2006. Fast approximated sift. In: Computer Vision-ACCV 2006, Springer, pp. 918927.

Jiang, Y., Xu, Y. and Liu, Y., 2013. Performance evaluation of feature detection and matching in stereo visual odometry. Neurocomputing 120, pp. 380-390.

Juan, L. and Gwun, O., 2009. A comparison of sift, pca-sift and surf. International Journal of Image Processing (IJIP) 3(4), pp. 143-152.

Khan, N. Y., McCane, B. and Wyvill, G., 2011. Sift and surf performance evaluation against various image deformations on benchmark dataset. In: Digital Image Computing Techniques and Applications (DICTA), 2011 International Conference on, IEEE, pp. 501-506.

Kitt, B., Geiger, A. and Lategahn, H., 2010. Visual odometry based on stereo image sequences with ransac-based outlier rejection scheme. In: Intelligent Vehicles Symposium (IV), 2010 IEEE, pp. 486-492.

Kneip, L., Scaramuzza, D. and Siegwart, R., 2011. A novel parametrization of the perspective-three-point problem for a direct computation of absolute camera position and orientation. In: Computer Vision and Pattern Recognition (CVPR), 2011 IEEE Conference on, IEEE, pp. 2969-2976.

Lowe, D. G., 2004. Distinctive image features from scaleinvariant keypoints. International journal of computer vision 60(2), pp. 91-110.

Luxen, M., 2003. Variance component estimation in performance characteristics applied to feature extraction procedures. In: Pattern Recognition, Springer, pp. 498-506.

Mikolajczyk, K. and Schmid, C., 2005. A performance evaluation of local descriptors. Pattern Analysis and Machine Intelligence, IEEE Transactions on 27(10), pp. 1615-1630.

Moulon, P., Monasse, P. and Marlet, R., 2013. Adaptive structure from motion with a contrario model estimation. In: Computer Vision-ACCV 2012, Springer, pp. 257-270.

Mouragnon, E., Lhuillier, M., Dhome, M., Dekeyser, F. and Sayd, P., 2006. Real time localization and 3d reconstruction. In: Computer Vision and Pattern Recognition, 2006 IEEE Computer Society Conference on, Vol. 1, IEEE, pp. 363-370.

Muja, M. and Lowe, D. G., 2009. Fast approximate nearest neighbors with automatic algorithm configuration. In: International Conference on Computer Vision Theory and Application VISSAPP'09), INSTICC Press, pp. 331-340.
Murillo, A. C., Guerrero, J. J. and Sagues, C., 2007. Surf features for efficient robot localization with omnidirectional images. In: Robotics and Automation, 2007 IEEE International Conference on, pp. 3901-3907.

Nistér, D., 2004. An efficient solution to the five-point relative pose problem. Pattern Analysis and Machine Intelligence, IEEE Transactions on 26(6), pp. 756-770.

Nistér, D., Naroditsky, O. and Bergen, J., 2004. Visual odometry. In: Computer Vision and Pattern Recognition, 2004. CVPR 2004. Proceedings of the 2004 IEEE Computer Society Conference on, Vol. 1, IEEE, pp. I-652.

Paparoditis, N., Papelard, J.-P., Cannelle, B., Devaux, A., Soheilian, B., David, N. and HOUZAY, E., 2012. Stereopolis ii: A multi-purpose and multi-sensor $3 \mathrm{~d}$ mobile mapping system for street visualisation and 3d metrology. Revue française de photogrammétrie et de télédétection (200), pp. 69-79.

Qu, X., Soheilian, B. and Paparoditis, N., 2015. Vehicle localization using mono-camera and geo-referenced traffic signs. In: 2015 IEEE Intelligent Vehicles Symposium (IV), pp. 605-610.

Saleem, S., Bais, A. and Sablatnig, R., 2012. A performance evaluation of sift and surf for multispectral image matching. In: Image Analysis and Recognition, Springer, pp. 166-173.

Schmid, C., Mohr, R. and Bauckhage, C., 2000. Evaluation of interest point detectors. International Journal of computer vision 37(2), pp. 151-172.

Schmidt, A., Kraft, M. and Kasiński, A., 2010. An evaluation of image feature detectors and descriptors for robot navigation. In: Computer Vision and Graphics, Springer, pp. 251-259.

Se, S., Lowe, D. and Little, J., 2001. Vision-based mobile robot localization and mapping using scale-invariant features. In: Robotics and Automation, 2001. Proceedings 2001 ICRA. IEEE International Conference on, Vol. 2, pp. 2051-2058 vol.2.

Strasdat, H., Montiel, J. M. and Davison, A. J., 2012. Visual slam: why filter? Image and Vision Computing 30(2), pp. 65-77.

Triggs, B., McLauchlan, P. F., Hartley, R. I. and Fitzgibbon, A. W., 2000. Bundle adjustmenta modern synthesis. In: Vision algorithms: theory and practice, Springer, pp. 298-372.

Valgren, C. and Lilienthal, A. J., 2010. Sift, surf \& seasons: Appearance-based long-term localization in outdoor environments. Robot. Auton. Syst. 58(2), pp. 149-156.

Yang, Y., Song, Y., Zhai, F., Fan, Z., Meng, Y. and Wang, J., 2009. A high-precision localization algorithm by improved sift key-points. In: Image and Signal Processing, 2009. CISP '09. 2nd International Congress on, pp. 1-6.

Zhang, Z., Deriche, R., Faugeras, O. and Luong, Q.-T., 1995. A robust technique for matching two uncalibrated images through the recovery of the unknown epipolar geometry. Artificial intelligence $78(1)$, pp. 87-119. 